\title{
Impact on survival through consolidation radiotherapy for diffuse large B-cell lymphoma: a comprehensive meta-analysis
}

\section{Martin D. Berger,${ }^{1 *}$ Sven Trelle, ${ }^{2 *}$ Annina E. Büchi, ${ }^{3}$ Sabrina Jegerlehner, ${ }^{3}$ Codruta Ionescu, ${ }^{4}$ Thierry Lamy de la Chapelle ${ }^{5}$ and Urban Novak ${ }^{1}$}

${ }^{1}$ Department of Medical Oncology, Inselspital, Bern University Hospital, University of Bern, Switzerland; ${ }^{2}$ CTU Bern, University of Bern, Switzerland; ${ }^{3}$ Department of General Internal Medicine, Inselspital, Bern University Hospital, University of Bern, Bern, Switzerland; ${ }^{4}$ Department of Radiation Oncology, Inselspital, Bern University Hospital, University of Bern, Switzerland and ${ }^{5}$ Hematology Department, Rennes University Hospital, INSERM Research Unit 1236, Rennes, France

*MDB and ST contributed equally as co-first authors.

\section{ABSTRACT}

$\mathrm{R}$ ituximab has improved response rates and overall survival in diffuse large B-cell lymphoma. Radiotherapy is an effective treatment - modality for lymphomas, but there is uncertainty on its use as consolidation after chemo-immunotherapy mainly in advanced stages. We evaluated its efficacy with a comprehensive meta-analysis and a systematic search of Pubmed, Embase, Cochrane, and abstracts from the American Society of Clinical Oncology, American Society of Hematology, European Society for Medical Oncology and American Society of Radiation Oncology published from June 1966 and December 2018. We identified 11 trials that evaluated consolidation radiotherapy following chemotherapy in a randomized fashion in 4,584 patients. The primary endpoint of this meta-analysis was progression-free survival (PFS). As three of the 11 trials were retracted, this data is based on 2,414 patients. For the primary endpoint, PFS, we found a hazard ratio (HR) 0.77 (95\% Confidence Interval [CI]: 0.51-1.17), pooled (tau ${ }^{2}: 0.25$; I': $85 \%$ ), and a HR 0.80 (95\% CI: $0.53-1.21$ ), pooled in a bivariate metaanalysis and for the secondary endpoint, overall survival, a HR 0.93 (range, 0.61-1.40), pooled (tau $\left.{ }^{2}: 0.25 ; \mathrm{I}^{2}: 74 \%\right)$ and a HR 0.86 (95\% CI: 0.58-1.27) in a bivariate meta-analysis. The lack of benefit did not change over time ( $P=0.95\left(\operatorname{tau}^{2}: 0.32 ; \mathrm{I}^{2}: 88 \%\right)$, and was also absent for PFS when stratifying for i) chemotherapy, ii) the use of rituximab, iii) age, iv) the dose of radiotherapy and $v$ ) application to patients in complete remission with bulky disease. None of the trials used a positron emission tomography-guided approach. This meta-analysis revealed no survival benefit when consolidation radiotherapy is given to unselected diffuse large Bcell lymphoma patients following chemotherapy. These results need to be considered in future trials in the positron emission tomography-computed tomography era.

\section{Introduction}

Comprising $35 \%$ of all non-Hodgkin lymphomas (NHL), diffuse large B-cell lymphoma (DLBCL) is the most common aggressive lymphoma in adults. The current standard therapy rituximab, cyclophosphamide, doxorubicin, vincristine, and prednisone (R-CHOP) cures two-thirds of patients. ${ }^{1,2}$ Several attempts with a variety of approaches including the addition of new drugs have so far failed to improve these results. ${ }^{3,4}$ Radiotherapy is an effective treatment option for patients with aggressive lymphomas. It was initially used as a primary modality for various lymphomas and was later used as consolidation when anthracycline-containing regimens became available in the 1980s. Radiotherapy is now commonly used in localized disease. ${ }^{5}$ As such, consolidation radiotherapy is part of the first line treat-

\section{Ferrata Storti Foundation}

Haematologica 2021

Volume 106(7):1923-1931

\section{Correspondence:}

URBAN NOVAK

urban.novak@insel.ch

Received: February 9, 2020.

Accepted: June 12, 2020.

Pre-published: June 18, 2020.

https://doi.org/10.3324/haematol.2020.249680

(C)2021 Ferrata Storti Foundation

Material published in Haematologica is covered by copyright. All rights are reserved to the Ferrata Storti Foundation. Use of published material is allowed under the following terms and conditions:

https://creativecommons.org/licenses/by-nc/4.0/legalcode. Copies of published material are allowed for personal or internal use. Sharing published material for non-commercial purposes is subject to the following conditions:

https://creativecommons.org/licenses/by-nc/4.0/legalcode, sect. 3. Reproducing and sharing published material for commercial purposes is not allowed without permission in writing from the publisher. 
ment of DLBCL in the European Society for Medical Oncology (ESMO) ${ }^{6}$ and National Comprehensive Cancer Network (NCCN) guidelines (https://www.nccn.org/professionals/physician_gls/pdf/b-cell.pdf (last access: Nov 28, 2019; for details see Online Supplementary Table S1). However, significant conceptual issues on its current use outside a clinical trial remain. They include different definitions of bulky disease, the use in advanced stages, and the recent implementation of positron emission tomography-computed tomography (PET-CT) in the clinical management. Albeit not limited to consolidation radiotherapy in DLBCL, treatment recommendations are often built on experience, clinical judgment and guidelines, but ideally should be based on data, preferably from randomized trials. Here we present a comprehensive meta-analysis to assess the impact of radiotherapy in addition to and after first-line chemo-immunotherapy of DLBCL based on the best currently available data by randomized controlled trials. With this large meta-analysis, we aim to provide the rational basis for a future randomized trial on the use of consolidation radiotherapy in DLBCL.

\section{Methods}

\section{Literature search}

We performed a comprehensive search in electronic databases (Pubmed, Embase, Cochrane) in any language between June 1966 and December 2018 for randomized controlled trials. As the data presented on meetings may differ from the peer-reviewed publications, ${ }^{7}$ a manual search was done of abstracts from ASCO, ASH, ESMO, and ASTRO proceedings between 2009 and 2018. We used the following search strategy: (radiation therap*[Title] OR radiotherapy*[Title] OR radio-therap*[Title]) AND (non-hodgkin*[Title] OR non Hodgkin*[Title] OR nonhodgkin[Title] OR no Hodgkin*[Title] OR nhl[Title]) OR (lymphoma*[Title]) AND

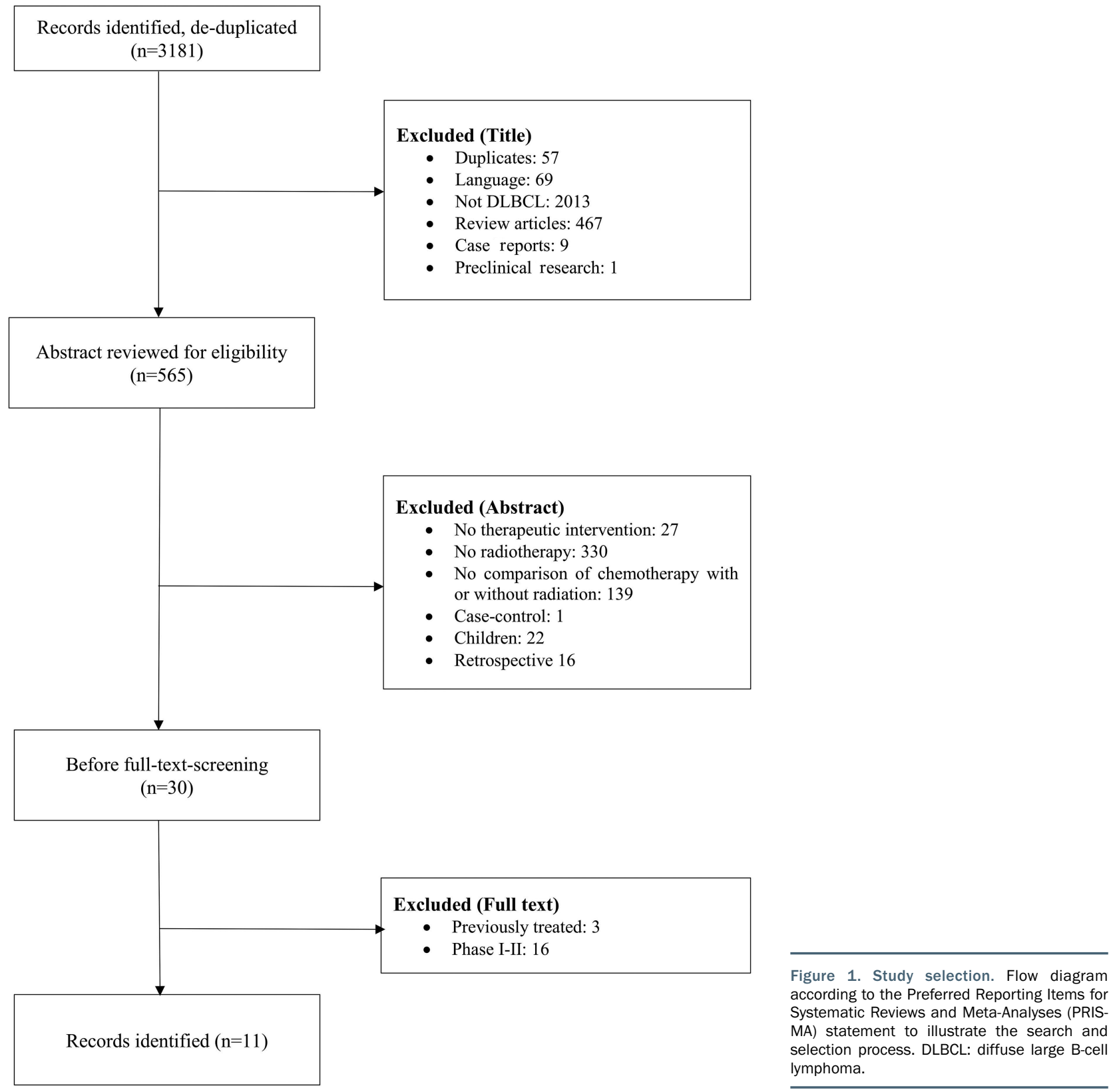


(aggressive[Title] OR malignant[Title] OR advanced[Title] OR histiocytic[Title] OR diffuse[Title] OR undifferentiated[Title] OR mixed[Title] OR high grade[Title] OR centroblastic [Title] OR immunoblastic[Title]).

\section{Inclusion criteria and trial selection}

Three investigators independently screened the studies. The flow diagram according to the PRISMA statement ${ }^{8,9}$ depicted in Figure 1 illustrates the search and selection process. We aimed at identifying randomized trials that had enrolled at least 50 adult patients ( $\geq 18$ years of age) per arm with newly diagnosed DLBCL (or aggressive lymphomas) at any stage according to the Ann Arbor classification. Patients had to be treated with a CHOP based chemotherapy (+/- rituximab), and randomized to subsequent consolidation radiotherapy or no radiotherapy. The 50 -patients cut-off was chosen to exclude therapeutic exploratory trials. Although the cut-off is arbitrary, it is safe to assume that no confirmatory trials were excluded given the high (progression-free) survival rates observed in this population. Patients with previously treated or relapsed DLBCL were excluded. The full text report of identified trials was independently checked by the three investigators. Disagreements regarding trial selection were discussed until consensus was found. Each report was scrutinized to eliminate duplicates and to ensure that it was published as an original article.

\section{Outcome measures}

Progression-free survival (PFS) and overall survival (OS) were the outcomes of interest. PFS was considered as tumor progression i.e., growth of the tumor during treatment, relapse i.e., growth after previous shrinkage or stabilization, or death. For trials that did not report outcome data that fit this definition, we used data of an outcome that was as close as possible to this definition e.g., event-free survival.

\section{Data extraction}

Data was extracted in duplicate and disagreements were resolved by consensus. We used the Cochrane 'Risk of Bias' approach to assess methodological quality of trials. ${ }^{10}$ We used the data from the original publications, from intention-to-treat analyses, and from randomized patients only, and for the longest follow-up available for a particular outcome. The hazard ratio (HR) was used as effect measure for both outcomes. If HR and a measure of precision (standard error, variance, or $95 \%$ Confidence Interval [CI]) was not available, we digitized Kaplan-Meier curves, reconstructed the underlying time-toevent data, and calculated (log) HR and standard errors using a Cox regression model. Details on the outcome data of the 11 trials are shown in Table 2.

\section{Statistical analysis}

Outcome data were pooled with a random-effects model using restricted maximum likelihood. We also did bivariate meta-analysis considering both outcomes in one analysis. Correlation between OS and PFS was estimated from two of the identified trials. ${ }^{11,12}$ We performed random-effects meta-regression for PFS over time using the mid of enrolment period as an independent covariate. Stratified analyses to explore possible reasons for heterogeneity were also done using meta-regression. Analyses were done using Stata (StataCorp. 2017. Stata Statistical Software: Release 16. College Station, TX, USA). Taking into account criticisms of meta-analysis, ${ }^{8,9}$ the Online Supplementary Appendix provides additional details on the analysis methods used and all outcome data. The latter, used in the meta-analysis, is provided in Table 2 .

\section{Results}

After deduplication, our search strategy generated 3,181 references (Figure 1). With the aim to identify clinical trials that assessed the role of consolidation radiotherapy in a randomized manner as part of the first-line therapy, our search revealed 11 trials amenable for this metaanalysis (details are listed in Table 1). Three of the four trials published by Aviles ${ }^{13-16}$ on this topic were later retracted. ${ }^{14-16}$ As of September 2019, these retracted papers have together received a total of 39 citations. Their data are provided in the respective figures, but were excluded from the meta-analyses. One trial was stopped early when the benefit of rituximab became evident, ${ }^{12}$ or as a result of a planned interim analysis. ${ }^{17}$ Older trials included lymphomas classified by the Kiel classification ${ }^{18}$ or included DLBCL according to the Working Formulation. ${ }^{19}$ Six of the trials included patients with localized disease only, but five of the 11 trials included also advanced stages. With the exception of the GELA LNH 93-1 ${ }^{11}$ where doxorubicin, cyclophosphamide, vindesine, bleomycin and prednisone (ACVBP) instead of $\mathrm{CHOP}$ was given in the comparator arm or SWOG, ${ }^{20}$ where the non-irradiated patients received eight cycles of CHOP (instead of three), the same chemotherapy was given to the randomized patients. The current standard $\mathrm{R}-\mathrm{CHOP}$ was used in four of the 11 trials. ${ }^{15-17,21,22}$ Only the recent Lysa/GOELAMS 0203 trial $^{21}$ used PET, although not for guided treatment. Radiotherapy was given to both localized stages 1 and 2, but also advanced disease, and either to all or only patients in complete remission or bulky disease. GOELAMS $0203^{21}$ was a non-inferiority trial whereas all other trials used for this meta-analysis used a superiority design.

Seven trials with a total of 2,488 patients contributed to the analysis of the primary endpoint PFS (Figure 2). Data were extracted from the original publications. The UNFOLDER trial was presented in part at the $12^{\text {th }}$ International Congress on Malignant Lymphomas, ${ }^{17}$ and again at the American Society of Clinical Oncology (ASCO) 2018, ${ }^{22}$ albeit with different endpoints. The latter have been used for this meta-analysis. Data from Engelhard ${ }^{18}$ was not available for the PFS analysis (Table 2). For PFS, the pooled HR was 0.77 (95\% CI: 0.51-1.17), and in the pooled bivariate meta-analysis HR was 0.80 (95\% CI: 0.53-1.21) (Figure 2). For OS, eight trials with a total of 2,744 patients were included. The pooled HR was 0.93 (95\% CI: 0.61-1.40) and 0.86 (95\% CI: 0.58-1.27) in the bivariate meta-analysis (Figure 3 ). Between-trial heterogeneity was high for both outcomes (PFS, tau ${ }^{2}: 0.25, \mathrm{I}^{2}$ : $85 \%$; OS, $\operatorname{tau}^{2}: 0.25, \mathrm{I}^{2}: 74 \%$ ). The total of 4,584 patients included in this meta-analysis were recruited between 1983 and 2013. However, the lack of benefit of the combined treatment modality remained stable over time, and time alone cannot explain the observed heterogeneity in the meta-analysis $\left(P\right.$-value for time trend $=0.95$; $\operatorname{tau}^{2}: 0.32$; $I^{2}: 88 \%$; Figure 4$)$.

Given the significant heterogeneity (see also Table 1), we analyzed the data by using the following stratifications: i) the applied chemotherapy was similar in both arms, ii) whether rituximab was used, iii) the dose of radiotherapy, and iv) whether the radiotherapy dose was given only in complete morphologic remission. In addition, we stratified according to the following trial population characteristics: v) mean age of the treated patients, 
vi) whether the majority had advanced stage, and vii) whether the majority had bulky disease. As shown in Figure 5, we failed to explain between-trial heterogeneity by stratifying on any of these subgroups.

\section{Discussion}

We here provide a large and comprehensive metaanalysis with the best currently available data from randomized trials on consolidation radiotherapy in the firstline treatment of aggressive lymphomas. In summary, we find no evidence for a survival benefit of an unselected consolidation radiotherapy for these patients, but uncertainty remains high.

Our analysis extends the data from both retrospective and uncontrolled series in favor ${ }^{2,23-25}$ or against ${ }^{26}$ the use of consolidation radiotherapy in the first line setting. Our state-of-the art and updated meta-analysis that takes into account general concerns on the reproducibility of metaanalysis 8,9 and significantly corroborates a previous metaanalysis on a limited number of trials. ${ }^{27}$ It also goes beyond extrapolations from data on particular extranodal sites ${ }^{28}$ the common use of consolidation radiotherapy for limited clinical stages only, ${ }^{23}$ pretreatment with different chemotherapy, ${ }^{29}$ or to treat bulky disease only. ${ }^{2}$ Collectively, the latter data are the basis for the current recommendations on the combined treatment modality also for patients with advanced stages. They have created an unsatisfactory uncertainty and rely on experts' opinions on the use of radiotherapy when facing an individual patient. However, DLBCL is a disease in which cure, but also treatment-related toxicities and economic factors have to be considered. Unfortunately, our meta-analysis cannot provide data on costs, safety and long term risks of secondary malignancies related to radiation therapy.

Overall, the data that could be used for this meta-analysis is of mixed quality (Tables 1 and 2). As an extreme, three of the four randomized trials by the same group all clearly supporting the added value of radiotherapy have later been retracted, the last one in early $2019 .{ }^{14-16} \mathrm{We}$ display their results in our figures as they might have influenced the use of consolidation radiotherapy in routine practice or clinical trials before their retraction. The results of the important UNFOLDER trial is still not fully published. ${ }^{17,22}$ The trials used for this meta-analysis also harbor considerable conceptual heterogeneity: radiotherapy was given to shorten chemotherapy and its toxicity,

Table 1. Summary on the randomized trials used for the meta-analysis. The number of patients in the respective column indicates the actual number of patients for the individual trials that received consolidation radiotherapy in a randomized fashion. The retracted trials are highlighted in grey. The superscript number in the study column refers to the number of the references in the manuscript.

\begin{tabular}{|c|c|c|c|c|c|c|c|}
\hline $\begin{array}{l}\text { Trial } \\
\text { (with reference) }\end{array}$ & Diagnosis & Patients (\#) & $\begin{array}{l}\text { Recruitment } \\
\text { period }\end{array}$ & Mean age $(y)$ & $\begin{array}{l}\text { Same } \\
\text { chemotherapy } \\
\text { in both arms }\end{array}$ & $\begin{array}{l}\text { Rituximab } \\
\text { used }\end{array}$ & $\begin{array}{c}\text { Radiation } \\
\text { dose } \\
>30 \mathrm{~Gy}\end{array}$ \\
\hline Aviles et $a l^{13}$ & DLCL & 218 & 1983-1988 & $59-61$ & yes & no & yes \\
\hline Engelhard et al. ${ }^{18}$ & high grade NHL & 110 (of 548$)$ & 1986-1989 & 56 & yes & no & yes \\
\hline ECOG $1484^{19}$ & diffuse aggressive NHL & 172 (of 399) & 1984-1992 & 59 & yes & no & no \\
\hline Avileset al. ${ }^{14}$ & DLCL & 341 & 1989-1995 & $53-57$ & yes & no & yes \\
\hline SWOG $8736^{20}$ & intermediate \& high grade NHL & 401 (of 442 ) & 1988-1995 & 59 & no & no & yes \\
\hline GELA 93-1"11 & aggressive NHL & 318 (of 647 ) & 1993-2000 & $46-47$ & no & no & yes \\
\hline GELA $93-4^{12}$ & aggressive NHL & 576 & 1993-2002 & $68-69$ & yes & no & yes \\
\hline Aviles et $a l^{15}$ & PMBL & 124 (of 182$)$ & 2001-2004 & $32-35$ & yes & yes & no \\
\hline Aviles et al. ${ }^{16}$ & DLBCL & 258 (of 612 ) & $2006-2010$ & 53 & yes & yes & no \\
\hline UNFOLDER $^{17,22}$ & Largely DLBCL & 285 & 2005-2012 & 44 & no & yes & yes \\
\hline GOELAMS $0203^{21}$ & DLBCL & 334 & 2005-2013 & 56 & yes & yes & yes \\
\hline
\end{tabular}

\begin{tabular}{|c|c|c|c|c|}
\hline Iîial & Publication & Stages & Bullky disease & Randomized \\
\hline Aviles et al..$^{13}$ & Int J Radiat Biol 1994 & advanced & all & only CR and bulky disease \\
\hline Engelhard et al..$^{18}$ & Ann Oncol 1991 & localized \& advanced & 19\% initially; bulky not randomized & only CR pts \\
\hline ECOG $1484^{19}$ & J Clin Oncol 2004 & localized & $31 \%$ initially (tumor $>10 \mathrm{~cm}$ ) & only CR pts \\
\hline Aviles et al. ${ }^{14}$ & Leuk Lymphoma 2004 & advanced & all & only CR and bulky disease \\
\hline SWOG $8736^{20}$ & New Engl J Med 1998 & localized & number unknown, some initially & all \\
\hline GELA 93-1"11 & New Engl J Med 2005 & localized & $12 \%$ of RT pts.; $10 \%$ of non-RT pts & all \\
\hline GELA 93-4 $4^{12}$ & J Clin Oncol 2007 & localized & $9 \%$ of RT pts; $8 \%$ of non-RT pts. & all \\
\hline Aviles et al. ${ }^{15}$ & Int J Radiat Biol 2012 & localized & $94 \%$ of RT pts. & only CR pts \\
\hline Aviles et al. ${ }^{16}$ & Hematology 2018 & advanced & $30 \%$ of RT pts. & only CR and bulky disease \\
\hline UNFOLDER $^{17,22}$ & $\begin{array}{r}\text { (12-ICML;a122); } \\
\text { ASCO 2018;a7574 }\end{array}$ & localized \& advanced & $76 \%$ initially & $\begin{array}{l}\text { initially } 4 \text { arms; random } \\
\text { for RT only in CR pts. }\end{array}$ \\
\hline GOELAMS $0203^{21}$ & Blood 2018 & localized & for non-bulky disease only & $\begin{array}{c}\text { random at start, some PR pts. } \\
\text { received RT }\end{array}$ \\
\hline
\end{tabular}

NHL: non-Hodgkin lymphoma; DLBCL: diffuse large B-cell lymphoma; CR: complete response; pts: points; RT: radiotherapy; y: years. 
to improve the outcome of the first-line treatment ${ }^{11,12,17,22}$ or as a salvage option for patients who achieved only a partial remission after chemotherapy..$^{14,21}$ As the Korean "ASPIRE" trial was unfortunately later withdrawn (clinicaltrials gov. Indentifier: NCT02054559; three times R$\mathrm{CHOP}+$ radiotherapy $v s$. six times $\mathrm{R}-\mathrm{CHOP}$ for stage 1 and 2 DLBCL), there is currently no randomized trial supporting the widely used, recently updated and safe approach to give less chemotherapy and PET-guided radiotherapy to patients with localized DLBCL. ${ }^{5,23,30-33}$ Also the data on limited stage DLBCL which accounts for 30 $\%$ of the cases, harbor significant variability as different definitions for limited stage, bulky disease as well as risk stratification and extrapolations were used. ${ }^{2,34}$ This ren- ders the integration of all available results difficult. Furthermore, a detailed view goes beyond the possibilities of a meta-analysis analyzing population level data. Although we do not have information on the stage-modified-IPI20, ${ }^{35}$ for all trials included in our analysis, we assume that many patients with localized disease of this meta-analysis had a low risk disease. They have an excellent prognosis, regardless of radiotherapy. ${ }^{36}$ The FLYER trial established four cycles of R-CHOP to be sufficient for patients with favorable risk (and non-bulky) DLBCL. ${ }^{37}$ Radiotherapy in this trial was limited to the contralateral testis in case of testicular involvement. In the yet unpublished OPTIMAL $>60$ trial (clinicaltrials gov. Identifier: NCT014778542), radiotherapy (and two additional cycles

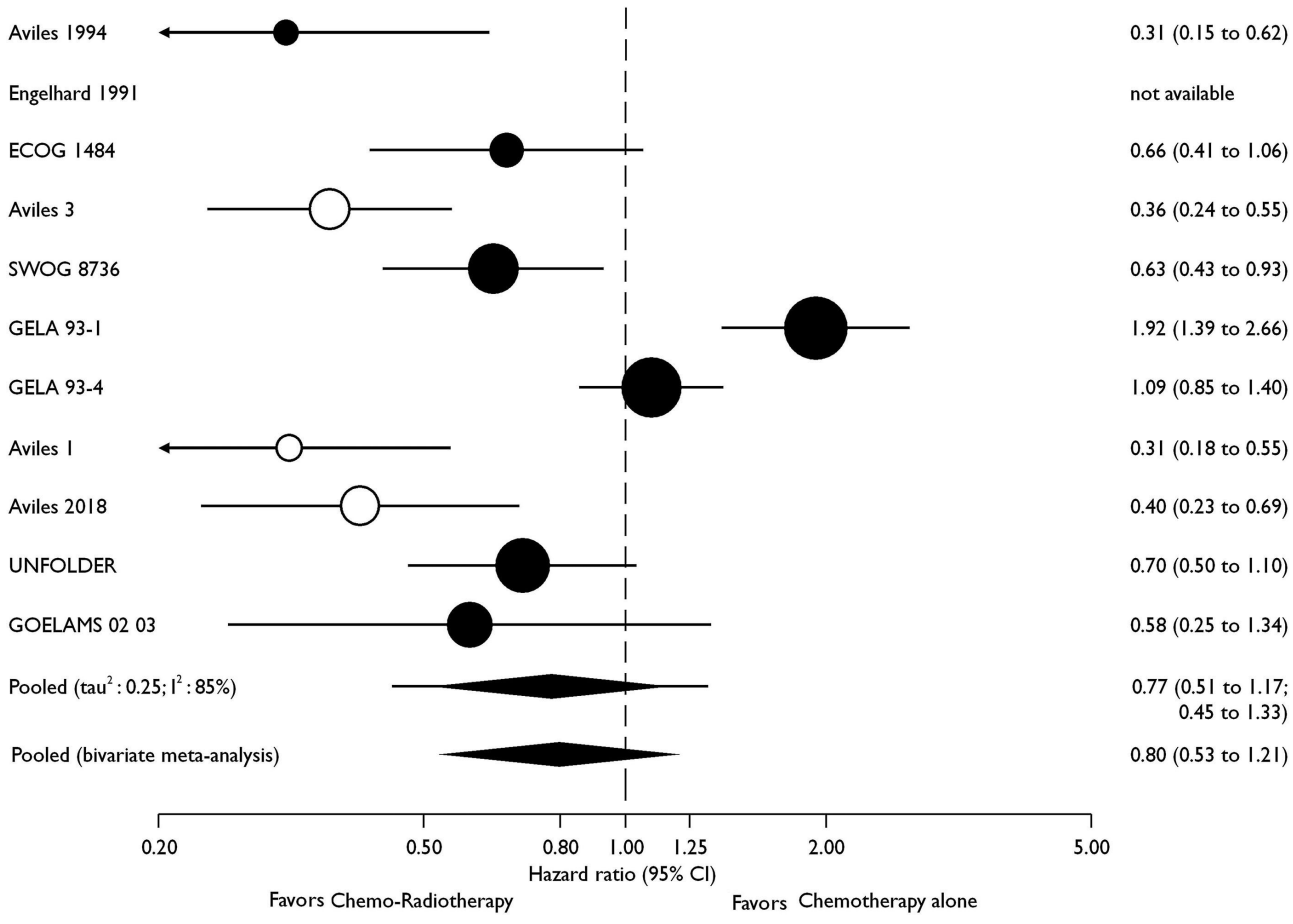

Figure 2. Effect of consolidation radiotherapy on progression-free survival. Circles are proportional to trial size i.e., number of patients; retracted trials are displayed with hollow circles.

Table 2. Outcome data of the individual trials used for the meta-analysis. Correlation between progression-free and overall survival were done for the GELA trial. The superscript number in the study column refers to the number of the references in the manuscript.

\begin{tabular}{|c|c|c|c|c|}
\hline $\begin{array}{l}\text { Trial } \\
\text { (with reference) }\end{array}$ & $\begin{array}{l}\text { Overall survival } \\
\text { Hazard ratio }\end{array}$ & $\begin{array}{c}\text { Progression-free survival } \\
\text { In HR (SE) }\end{array}$ & Hazard ratio & In HR (SE) \\
\hline Aviles et $a l^{13}$ & 0.33 & $-1.11(0.44)$ & 0.31 & ?-1.17 (0.36) \\
\hline Engelhard et al. ${ }^{18}$ & 2.09 & $0.74(0.58)$ & $\mathrm{n} / \mathrm{a}$ & $\mathrm{n} / \mathrm{a}$ \\
\hline ECOG $1484^{19}$ & 0.81 & $-0.21(0.28)$ & 0.66 & $?-0.41(0.24)$ \\
\hline Aviles et al. ${ }^{14}$ & 0.35 & $-1.04(0.25)$ & 0.36 & $-1.02(0.22)$ \\
\hline SWOG $8736^{20}$ & 0.64 & $-0.44(0.23)$ & 0.63 & $-0.46(0.19)$ \\
\hline GELA 93-1"11 & 1.98 & $0.68(0.20)$ & 1.92 & $0.65(0.16)$ \\
\hline GELA 93-4 ${ }^{12}$ & 1.08 & $0.07(0.14)$ & 1.09 & $0.09(0.13)$ \\
\hline Aviles et al..$^{15}$ & 0.21 & $-1.54(0.29)$ & 0.31 & $-1.16(0.28)$ \\
\hline Aviles et al. ${ }^{16}$ & 0.28 & $-1.27(0.32)$ & 0.4 & $-0.92(0.28)$ \\
\hline UNFOLDER $^{1,22}$ & 1.2 & $0.18(0.38)$ & 0.7 & $-0.36(0.20)$ \\
\hline GOELAMS $0203^{21}$ & 0.52 & $-0.66(0.45)$ & 0.58 & $-0.54(0.43)$ \\
\hline
\end{tabular}

HR: hazard ratio; ln: natural logarithm; SE: standard error. 
of chemotherapy) is given just to PET-positive sites after four cycles of chemotherapy. According to an interim analysis, this can compensate the inferior outcome of this population. ${ }^{37}$ Furthermore, the authors of this trial communicated that radiotherapy to PET-negative bulky disease is not needed. ${ }^{38}$
In the latest ESMO guidelines, consolidation radiotherapy for DLBCL patients is recommended for both elderly and intermediate- and high-risk young patients with bulky disease. ${ }^{6} \mathrm{NCCN}$ is less firm, and mainly restricts its recommendation to residual disease (partial remission or PET-positivity, Online Supplementary Table S1). These rec-

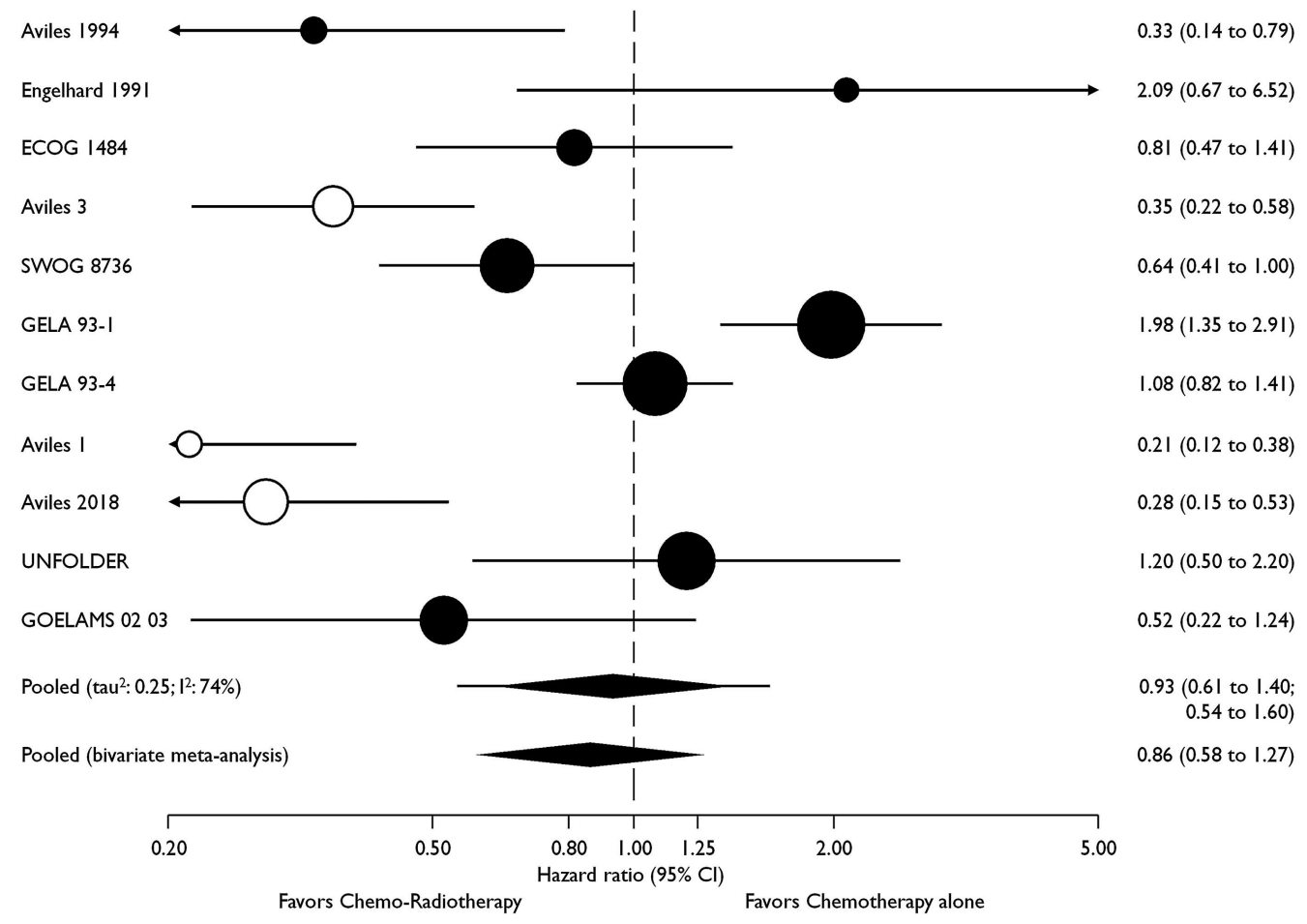

Figure 3. Effect of consolidation radiotherapy on overall survival. Circles are proportional to trial size i.e., number of patients; retracted trials are displayed with hollow circles. Cl: Confidence Interval.

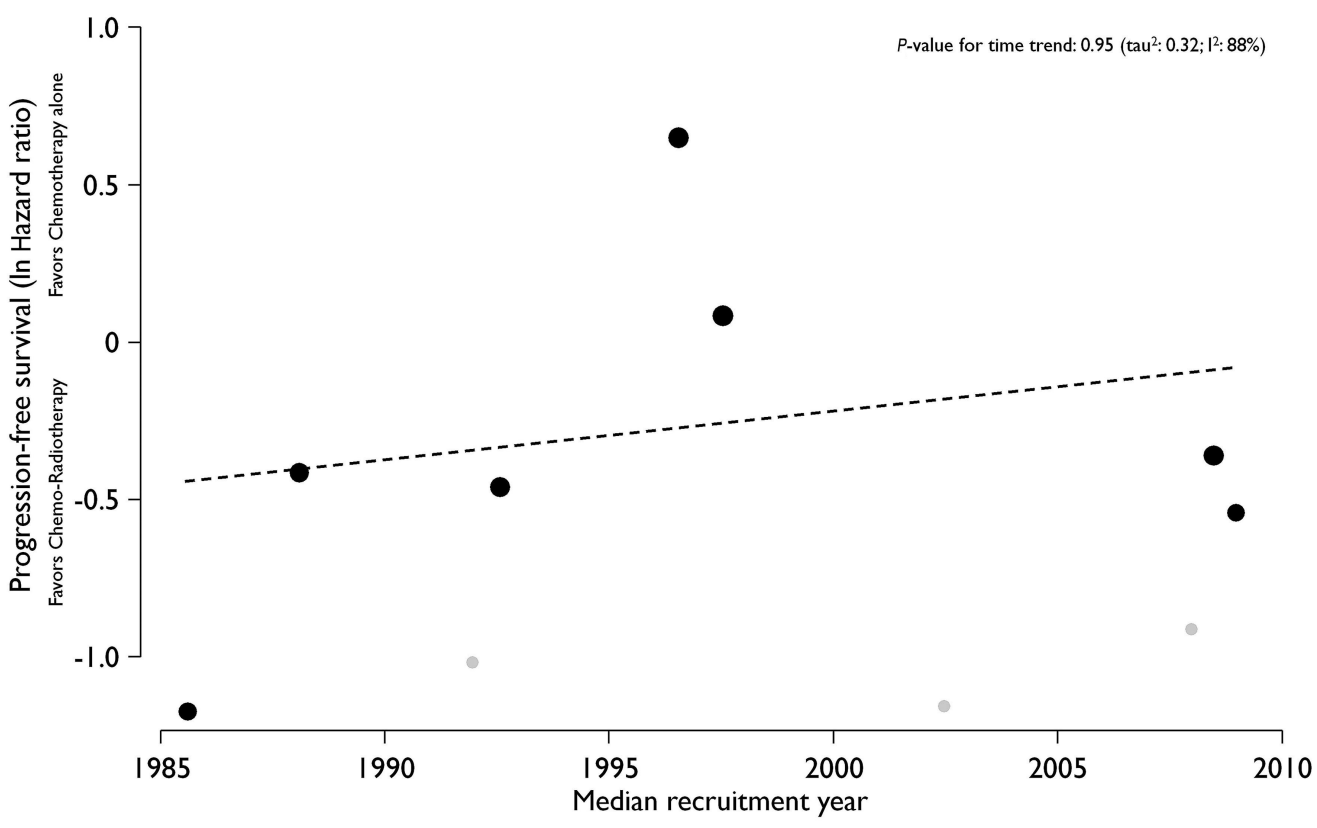

Figure 4. Time trend plot on the effect of consolidation radiotherapy. Circles are proportional to weight in analysis; dashed line shows the fitted linear regression; retracted trials are in grey. In: natural logarithm. 
ommendations are not fully supported by the results of this meta-analysis, especially by the results of the stratified analysis provided in Figure 5. The International Lymphoma Radiation Oncology Group (ILROG) has recently updated its guidelines, albeit in the relapsed and refractory setting. ${ }^{39}$ The trials analyzed in our meta-analysis did not specifically include patients with extranodal DLBCL for which both $\mathrm{ESMO}^{40}$ and ILROG ${ }^{41}$ have published separate guidelines. Specifically, consolidative mediastinal radiotherapy is currently recommended in responding primary mediastinal B-cell lymphoma (PMBL) patients after treatment with standard-dose chemoimmunotherapy. ${ }^{40}$ However, extrapolation of the data of our meta-analysis on DLBCL not otherwise specified (NOS) to and from entities such as primary mediastinal lymphoma, primary central nervous system (CNS) or testicular lymphoma is discouraged. The safe omission of whole brain radiotherapy for CNS lymphomas is conceptually controversial. ${ }^{42,43}$ As the role of adjuvant mediastinal radiotherapy in $\mathrm{PMBL}$ patients with complete remission after chemotherapy is unclear and a large number of patients are cured by chemotherapy alone with DAEPOCH-R,${ }^{44}$ it is important to note that accrual in IELSG37 (clinicaltrials gov. Identifier: NCT01599559) has recently been completed; this potentially practice changing randomized trial with a non-inferiority design has evaluated the role of consolidation radiotherapy in PETnegative patients.

Our meta-analysis provides further evidence that patients with a complete morphologic remission after chemotherapy or initial bulky disease are unlikely to particularly profit from consolidation radiotherapy. ${ }^{25,38}$ PET has become an integral part of the treatment of DLBCL patients, although the prognostic value of interim PET is limited, ${ }^{45,46}$ and a PET-based escalation of chemotherapy was unable to improve the outcome. ${ }^{47}$ None of the trials that we included in our meta-analysis used a truly PETguided treatment approach. This was applied in limited stage DLBCL in a retrospective ${ }^{32}$ and also a prospective, ${ }^{30}$ albeit non-randomized trial. In order not to add also radiotherapy to the recent painful flaws in clinical DLBCL research, 3,4 our meta-analysis should be taken into account when a new trial is planned. We provide evidence on patients that we should rather not selectively irradiate, but we still do not know how to use consolidation radiotherapy. Besides its wide and established use in localized disease, ${ }^{5}$ we see the rationale use of radiotherapy in DLBCL patients analogous to the current situation in Hodgkin's disease, e.g., for insufficient responses to chemo-immunotherapy. Considering retrospective trials, ${ }^{38,48}$ radiotherapy could be restricted to PET-positive rests. Among other unanswered questions, this would be practice changing. Ideally, this hypothesis needs corroboration in two separate prospective trials to randomly apply radiotherapy in trial 1 for patients with PET-negative, and trial 2 for patients with PET-positive rests. The first trial would be a non-inferiority trial to proof whether it is safe to not irradiate patients perceived

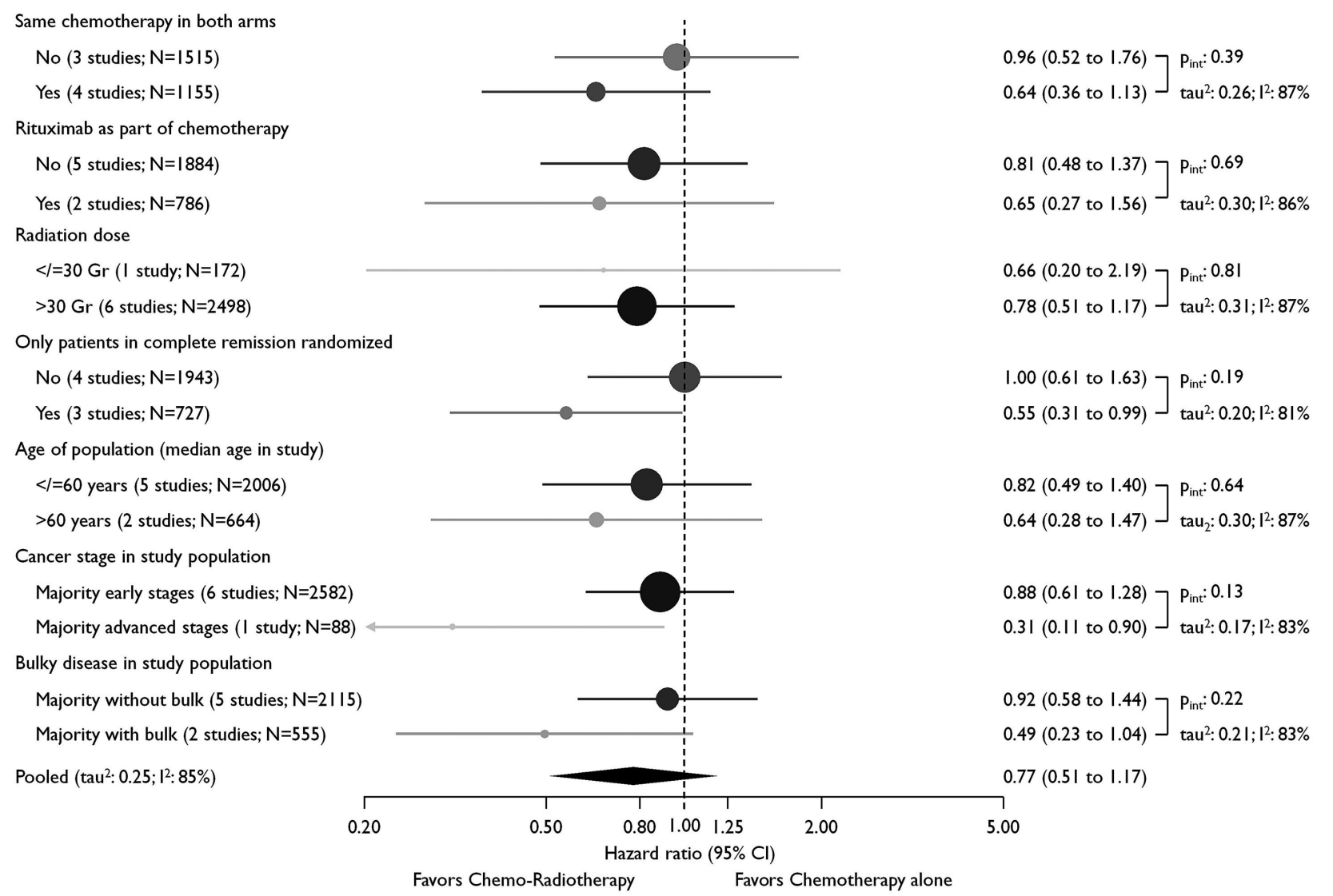

Figure 5. Stratified progression free survival analysis on the effect of consolidation radiotherapy. Circles are proportional to stratum size i.e., overall number of patients in stratum; color of circles reflects number of trials in stratum i.e., from black (seven trials) to light grey (one trial); age, stage, and bulky disease are characteristics of the study population and cannot be interpreted on the individual participant level (ecological fallacy). Cl: Confidence Interval. 
to be cancer-free. Trial 2 would be a superiority design testing whether radiotherapy is able to improve the outcome residual DLBCL after chemo-immunotherapy. Assuming a 2-year PFS, an appropriate and pragmatic endpoint in DLBCL ${ }^{49,50}$ of $80 \%$, an alpha of 0.025 for the non-inferiority (one-sided) and 0.05 (two-sided) for the superiority trial, a power of $80 \%$ and enrolment over 5 years, we calculated the following sample size: trial 1 (failure rate of $24 \%$ [HR 1.23]), would require 1,916 overall or 384 patients per year; trial 2 (and a HR of 0.75 or an improvement of the 2-year PFS to $85 \%$ ) would need 1,098 patients or 220 patients per year. Assuming an endof-therapy PET-positivity of $25-30 \%,{ }^{45} 4,000$ or 5,000 patients respectively have to be screened. Clearly, such numbers need a global and fully committed academic effort. However, otherwise the important question on the role of consolidation radiotherapy in DLBCL, which with the current data, regularly gives rise to unsatisfactory and futile discussions at lymphoma boards, will never be answered convincingly. Based on this meta-analysis and other data, ${ }^{21}$ we favor a superiority trial that first allocates a role of consolidation radiotherapy in DLBCL. Then, one may also test the use of smaller irradiation volumes according to the concept of involved node ver- sus involved site radiotherapy using modern techniques (intensity modulated radiotherapy [IMRT]) to reduce doses to organs at risk. ${ }^{31,51} \mathrm{New}$ trials could also approach unanswered questions on the role of consolidation radiotherapy in other subpopulations like patients with interim PET positive disease, or in limited stage disease of high risk histologies such as double hit lymphomas although the prognosis of the later may be better than previously perceived. ${ }^{52}$

\section{Disclosures}

No conflicts of interest to disclose.

\section{Contributions}

$M D B$ performed research, analyzed data and wrote parts of the paper; ST analyzed data, contributed vital material and wrote parts of the paper; $A E B$ and SJ performed research and analyzed data; CI analyzed data; TL contributed vital material; UN had the idea, designed research, analyzed data, contributed vital material, and wrote the paper.

\section{Acknowledgments}

We thank Doris Kopp for the literature search, as well as Matthias Egger and Emanuele Zucca for valuable comments.

\section{References}

1. Coiffier B, Thieblemont C, Van Den Neste $\mathrm{E}$, et al. Long-term outcome of patients in the LNH-98.5 trial, the first randomized study comparing rituximab-CHOP to standard CHOP chemotherapy in DLBCL patients: a study by the Groupe d'Etudes des Lymphomes de l'Adulte. Blood. 2010;116(12):2040-2045

2. Pfreundschuh M, Kuhnt E, Trumper L, et al. CHOP-like chemotherapy with or without rituximab in young patients with goodprognosis diffuse large-B-cell lymphoma: 6year results of an open-label randomised study of the MabThera International Trial (MInT) Group. Lancet Oncol. 2011;12(11): 1013-1022.

3. Iacoboni G, Zucca E, Ghielmini M, Stathis A. Methodology of clinical trials evaluating the incorporation of new drugs in the firstline treatment of patients with diffuse large B-cell lymphoma (DLBCL): a critical review. Ann Oncol. 2018;29(5):1120-1129.

4. Goy A. Succeeding in Breaking the R-CHOP Ceiling in DLBCL: learning from negative trials. J Clin Oncol. 2017;35(31):3519-3522.

5. Persky DO, Unger JM, Spier CM, et al. Phase II study of rituximab plus three cycles of $\mathrm{CHOP}$ and involved-field radiotherapy for patients with limited-stage aggressive Bcell lymphoma: Southwest Oncology Group study 0014. J Clin Oncol. 2008;26(14):22582263.

6. Tilly H, Gomes da Silva M, Vitolo U, et al. Diffuse large B-cell lymphoma (DLBCL): ESMO Clinical Practice Guidelines for diagnosis, treatment and follow-up. Ann Oncol. 2015;26(Suppl 5):v116-125.

7. Beyar-Katz O, Rowe JM, Townsend LE, Tallman MS, Hadomi R, Horowitz NA. Published abstracts at international meetings often over- or underestimate the initial response rate. Blood. 2017;129(16):23262328.

8. Wayant C, Page MJ, Vassar M. Evaluation of reproducible research practices in oncology systematic reviews with meta-analyses referenced by National Comprehensive Cancer Network Guidelines. JAMA Oncol. 2019;5(11):1550-1555.

9. Unger JM. Reproducible findings in systematic reviews and meta-analyses in oncology: verify, then trust. JAMA Oncol. 2019;5(11): 1545-1546.

10. Higgins JP, Altman DG, Gotzsche PC, et al. The Cochrane Collaboration's tool for assessing risk of bias in randomised trials. BMJ. 2011;343:d5928.

11. Reyes F, Lepage E, Ganem G, et al. ACVBP versus $\mathrm{CHOP}$ plus radiotherapy for localized aggressive lymphoma. N Engl J Med. 2005;352(12):1197-1205.

12. Bonnet C, Fillet G, Mounier N, et al. CHOP alone compared with $\mathrm{CHOP}$ plus radiotherapy for localized aggressive lymphoma in elderly patients: a study by the Groupe d'Etude des Lymphomes de l'Adulte. J Clin Oncol. 2007;25(7):787-792.

13. Aviles A, Delgado S, Nambo MJ, Alatriste S, Diaz-Maqueo JC. Adjuvant radiotherapy to sites of previous bulky disease in patients stage IV diffuse large cell lymphoma. Int J Radiat Oncol Biol Phys. 1994;30(4):799-803.

14. Aviles A, Fernandez R, Perez F, et al. Adjuvant radiotherapy in stage IV diffuse large cell lymphoma improves outcome. Leuk Lymphoma. 2004;45(7):1385-1389.

15. Aviles A, Neri N, Fernandez R, HuertaGuzman J, Nambo MJ. Randomized clinical trial to assess the efficacy of radiotherapy in primary mediastinal large B-lymphoma. Int J Radiat Oncol Biol Phys. 2012;83(4):12271231

16. Aviles A, Nambo MJ, Calva A, Neri N, Cleto S, Silva L. Retracted article: adjuvant radiotherapy in patients with diffuse large B-cell lymphoma in advanced stage (III/IV) improves the outcome in the rituximab era. Hematology. 2019;24(1):521-525.

17. Zwick C, Held G, Ziepert M, et al. The role of radiotherapy to bulky disease in elderly patients with aggressive B-cell lymphoma. Results from two prospective trials of the
DSHNHL. Hematol Oncol. 2013;31(Suppl 1):S96-150.

18. Engelhard M, Meusers P, Brittinger G, et al. Prospective multicenter trial for the response-adapted treatment of high-grade malignant non-Hodgkin's lymphomas: updated results of the COP-BLAM/IMVP-16 protocol with randomized adjuvant radiotherapy. Ann Oncol. 1991;2(Suppl 2):S177180

19. Horning SJ, Weller E, Kim K, et al. Chemotherapy with or without radiotherapy in limited-stage diffuse aggressive nonHodgkin's lymphoma: Eastern Cooperative Oncology Group study 1484. J Clin Oncol. 2004;22(15):3032-3038.

20. Miller TP, Dahlberg S, Cassady JR, et al. Chemotherapy alone compared with chemotherapy plus radiotherapy for localized intermediate- and high-grade nonHodgkin's lymphoma. N Engl J Med. 1998;339(1):21-26.

21. Lamy T, Damaj G, Soubeyran P, et al. RCHOP 14 with or without radiotherapy in nonbulky limited-stage diffuse large B-cell lymphoma. Blood. 2018;131(2):174-181.

22. Pfreundschuh M, Murawski N, Ziepert M et al. Radiotherapy (RT) to bulky (B) and extralymphatic (E) disease in combination with $6 \times \mathrm{R}-\mathrm{CHOP}-14$ or R-CHOP-21 in young good-prognosis DLBCL patients: Results of the $2 \times 2$ randomized UNFOLDER trial of the DSHNHL/GLA. J Clin Oncol. 2018;360(Suppl 15):S7574.

23. Vargo JA, Gill BS, Balasubramani GK Beriwal S. Treatment selection and survival outcomes in early-stage diffuse large B-cell lymphoma: do we still need consolidative radiotherapy? J Clin Oncol. 2015;33(32): 3710-3717.

24. Dabaja BS, Vanderplas AM, CrosbyThompson AL, et al. Radiation for diffuse large B-cell lymphoma in the rituximab era: analysis of the National Comprehensive Cancer Network lymphoma outcomes project. Cancer. 2015;121(7):1032-1039.

25. Held G, Murawski N, Ziepert M, et al. Role 
of radiotherapy to bulky disease in elderly patients with aggressive B-cell lymphoma. J Clin Oncol. 2014;32(11):1112-1118.

26. Casasnovas RO, Ysebaert L, Thieblemont C, et al. FDG-PET-driven consolidation strategy in diffuse large B-cell lymphoma: final results of a randomized phase 2 study. Blood. 2017;130(11):1315-1326.

27. dos Santos LV, Lima JP, Lima CS, Sasse EC, Sasse $\mathrm{AD}$. Is there a role for consolidative radiotherapy in the treatment of aggressive and localized non-Hodgkin lymphoma? A systematic review with meta-analysis. BMC Cancer. 2012;12:288.

28. Held G, Zeynalova S, Murawski N, et al. Impact of rituximab and radiotherapy on outcome of patients with aggressive B-cell lymphoma and skeletal involvement. J Clin Oncol. 2013;31(32):4115-4122.

29. Recher C, Coiffier B, Haioun C, et al. Intensified chemotherapy with ACVBP plus rituximab versus standard $\mathrm{CHOP}$ plus rituximab for the treatment of diffuse large B-cell lymphoma (LNH03-2B): an open-label randomised phase 3 trial. Lancet. 2011;378 (9806):1858-1867.

30. Persky DO, Li H, Stephens DM, et al. PETdirected therapy for patients with limitedstage diffuse large B-cell lymphoma - results of Intergroup NCTN Study S1001. Blood. 2019;134(Suppl 1):S349.

31. Campbell BA, Connors JM, Gascoyne RD, Morris WJ, Pickles T, Sehn LH. Limitedstage diffuse large B-cell lymphoma treated with abbreviated systemic therapy and consolidation radiotherapy: involved-field versus involved-node radiotherapy. Cancer. 2012;118(17):4156-4165

32. Sehn LH, Scott DW, Villa D, et al. Long-term follow-up of a PET-guided approach to treatment of limited-stage diffuse large B-cell lymphoma (DLBCL) in British Columbia (BC). Blood. 2019;134(Suppl 1):S401.

33. Sehn LH. Chemotherapy alone for localized diffuse large B-cell lymphoma. Cancer J. 2012;18(5):421-426.

34. Persky DO. Limited-stage DLBCL: it's patient selection. Blood. 2018;131(2):155-156.

35. A predictive model for aggressive nonHodgkin's lymphoma. The International Non-Hodgkin's Lymphoma Prognostic Factors Project. N Engl J Med. 1993;329
(14):987-994.

36. Miller TP. The limits of limited stage lymphoma. J Clin Oncol. 2004;22(15):2982 2984.

37. Poeschel V, Held G, Ziepert M, et al. Four versus six cycles of $\mathrm{CHOP}$ chemotherapy in combination with six applications of rituximab in patients with aggressive B-cell lymphoma with favourable prognosis (FLYER): a randomised, phase 3 , non-inferiority trial. Lancet. 2020;394(10216):2271-2281.

38. Pfreundschuh M, Christofyllakis K, Altmann $\mathrm{B}$, et al. Radiotherapy to bulky disease PET negative after immunochemotherapy can be spared in elderly DLBCL patients: results of a planned interim analysis of the first 187 patients with bulky disease treated in the OPTIMAL > 60 study of the DSHNHL. Clin Oncol. 2017;35(Suppl 15):S7506.

39. Ng AK, Yahalom J, Goda JS, et al. Role of radiation therapy in patients with relapsed/refractory diffuse large B-cell lymphoma: guidelines from the International Lymphoma Radiation Oncology Group. Int J Radiat Oncol Biol Phys. 2018;100(3):652669

40. Vitolo U, Seymour JF, Martelli M, et al Extranodal diffuse large B-cell lymphoma (DLBCL) and primary mediastinal B-cell lymphoma: ESMO Clinical Practice Guidelines for diagnosis, treatment and follow-up. Ann Oncol. 2016;27(Suppl 5):v91 v102.

41. Yahalom J, Illidge T, Specht L, et al. Modern radiation therapy for extranodal lymphomas: field and dose guidelines from the International Lymphoma Radiation Oncology Group. Int J Radiat Oncol Biol Phys. 2015;92(1):11-31.

42. Thiel E, Korfel A, Martus P, et al. High-dose methotrexate with or without whole brain radiotherapy for primary CNS lymphoma (G-PCNSL-SG-1): a phase 3, randomised non-inferiority trial. Lancet Oncol. 2010;11(11):1036-1047

43. Ferreri AJM, Cwynarski K, Pulczynski E, et al. Whole-brain radiotherapy or autologous stem-cell transplantation as consolidation strategies after high-dose methotrexatebased chemoimmunotherapy in patients with primary CNS lymphoma: results of the second randomisation of the International
Extranodal Lymphoma Study Group-32 phase 2 trial. Lancet Haematol. 2017;4(11) e510-e523.

44. Dunleavy K, Pittaluga S, Maeda IS, et al. Dose-adjusted EPOCH-rituximab therapy in primary mediastinal B-cell lymphoma. N Engl J Med. 2013;368(15):1408-1416.

45. Mamot C, Klingbiel D, Hitz F, et al. Final results of a prospective evaluation of the predictive value of interim positron emission tomography in patients with diffuse large B-cell lymphoma treated with $\mathrm{R}$ CHOP-14 (SAKK 38/07). J Clin Oncol. 2015;33(23):2523-2529.

46. Kurtz DM, Scherer F, Jin MC, et al Circulating tumor DNA measurements as early outcome predictors in diffuse large Bcell lymphoma. J Clin Oncol. 2018;36(28): 2845-2853.

47. Duhrsen U, Müller S, Hertenstein B, et al Positron emission tomography-guided therapy of aggressive non-Hodgkin lymphomas (PETAL): a multicenter, randomized phase III trial. J Clin Oncol. 2018;36(20):2024-2034

48. Freeman CF, Savage KJ, Villa D, et al. Longterm results of PET-guided radiation therapy in patients with advanced-stage diffuse large B-cell lymphoma treated with R-CHOP in British Columbia. Blood. 2017;130(Suppl 1):S823.

49. Maurer MJ, Habermann TM, Shi $\mathrm{O}$, et al. Progression-free survival at 24 month (PFS24) and subsequent outcome for patients with diffuse large B-cell lymphoma (DLBCL) enrolled on randomized clinical trials. Ann Oncol. 2018;29(8):1822-1827.

50. Shi O, Schmitz N, Ou FS, et al. Progressionfree Survival as a surrogate end point for overall curvival in first-line diffuse large $\mathrm{B}$ cell lymphoma: an individual patient-level analysis of multiple randomized trials (SEAL). J Clin Oncol. 2018;36(25):25932602.

51. Specht L, Yahalom J. The concept and evolution of involved site radiation therapy for lymphoma. Int J Clin Oncol. 2015;20(5):849854

52. Torka P, Kothari SK, Sundaram S, et al Outcomes of patients with limited-stage aggressive large B-cell lymphoma with highrisk cytogenetics. Blood Adv. 2020;4(2):253262. 